TUMOUR BIOLOGY Chemical Transformants

from our Cell Biology Correspondent

THE properties of chick embryo fibroblasts infected and transformed by certain temperature sensitive mutants of Rous sarcoma viruses have estaulished that the maintenance of the transformed cell phenotype depends upon the continuous expression of one or more viral functions. Similar experiments with a solitary temperature sensitive mutant of polyoma virus lead to the same conclusion. Both the RNA and the DNA tumour viruses have small genomes and it seems unlikely, to say the least, that each and every change in the phenotype of the cell transformed by one of these viruses results directly from the action of a distinct viral protein. The viruses simply do not have enough genetic information to be so profligate. It follows therefore that the limited number of transforming proteins specified by the tumour viruses must induce either pleiotropic effects or they must interact with various cellular molecules and indirectly bring about transformation.

At present the nature of the transforming viral proteins is not known, neither is anything known about the putative cellular molecules which may be targets for the viral proteins. But just as it is possible to inactivate by mutation the viral genes involved in transformation, it ought to be possible to mutate the cellular genes specifying the target molecules, and Di Mayorca et al. (Proc. US Nat. Acad. Sci., 70, 46 ; 1973) may have done this; certainly the data they present can be so interpreted.

$\mathrm{Di}$ Mayorca et al, exposed $\mathrm{BHK}_{21}$ clone 13 cells to dimethylnitrosoamine or nitrosomethylurea and then plated the cells in liquid media to measure their plating efficiency and in soft agar to measure their ability to grow in suspension into colonies. These BHK cells do not grow in agar suspension unless they have been transformed and this technique of suspension plating has been widely used as a method of selecting and assaying for transformation of BHK cells. Using these procedures Di Mayorca et al. detected transformants at a frequency of $6 \times 10^{-6}$ in populations of $\mathrm{BHK}$ cells exposed to appropriate doses of either dimethylnitrosamine or nitrosomethylurea. That transformants arise after exposure to these chemical carcinogens/mutagens is not in itself particularly surprising, but the phenotype of the transformed cells is remarkable. Apparently all the transformants isolated after the cells have been exposed to the carcinogens as well as a single clone of spontaneously transformed BHK cells prove to be temperature sensitive for the two markers of the transformed

state that were assayed-the ability to grow in agar suspension and the morphology of the colonies. In all cases these transformants failed to grow in agar at $32^{\circ} \mathrm{C}$ and in liquid media grew to form typical monolayers, but at $38.5^{\circ} \mathrm{C}$ they behaved as transformed cells growing to form colonies in agar and to form multilayered foci in liquid medium.

These results are apparently fully reproducible and the properties of these chemical transformants are in marked contrast to the properties of BHK cells transformed by polyoma virus which exhibit the transformed phenotype at $32^{\circ} \mathrm{C}$ and $38.5^{\circ} \mathrm{C}$. These temperature sensitive chemical transformants also differ from cells transformed by temperature sensitive tumour viruses, the latter having a transformed phenotype at lower temperatures and an untransformed phenotype at higher temperatures.

The obvious interpretation of these data which Di Mayorca et al. offer is that the cells they have isolated carry a temperature sensitive lesion in a cell gene which specifies a product required for the maintenance of the normal or untransformed phenotype. They suggest that at $32^{\circ} \mathrm{C}$ the putative cellular gene product is functional whereas at $38.5^{\circ} \mathrm{C}$ it is inactivated because of a temperature sensitive mutation and as a result the cells acquire the transformed phenotype as their temperature is raised. Clearly if such a cellular gene product, that is required to maintain a cell in the untransformed state, exists it must be inactivated when a cell is transformed by a tumour virus; it must presumably be inactivated, for example, when

\title{
Transformation by Herpesvirus
}

SOME herpesviruses of mammals are known to possess an oncogenic potential, and Duff and Rapp (Nature New Biology, 233, 48; 1971) believe that herpes simplex virus, a human virus, can transform hamster cells. Whether herpes simplex virus or any of the other herpesviruses which are indigenous to man have any role in the development of cancer in human beings is, however, another and a vexed question. Obviously human herpesviruses, which can be shown to transform human cells in culture, must be seriously considered as potential human carcinogens and for this reason numerous groups are carrying out the appropriate experiments. Darai and Munk in Heidelberg, for example, have been investigating the infection of human embryonic lung cells with Herpesvirus hominis type 2, and they report in next Wednesday's Nature New Biology (February 28) that when cultured in appropriate
BHK cells are transformed by polyoma virus and this putative cellular protein may be a target of the viral transforming gene proteins.

No doubt such speculations are not to everybody's taste, but surely few would deny that the cells which Di Mayorca and his colleagues have isolated have remarkable properties.

\section{TEMPERATE VIRUSES \\ Infection in Humans}

from our Medical Virology Correspondent Subacute sclerosing panencephalitis (SSPE), a fatal disease of children and young adults, is a chronic progressive infection of the central nervous system by a measles-like virus (M. Katz et al., Nature, 222, 888; 1969 ; and V. Ter Meulen et al., Curr. Topics Microbiol. Immunol., 57, 1 ; 1972). The evolution of the disease can be divided into clinical stages characterized by mental and behavioural changes, disorders of the locomotor system and loss of cerebral-cortex function. The SSPE agent has many of the biological properties of measles virus, but unlike conventional strains of measles it remains intimately bound to cells and it has a different pattern of replication and intracellular behaviour.

The chronic nature of this viral infection, the possibility that the formation of immune complexes may be important in the aetiology of this disease and perhaps in causing some of its damage to nervous tissue (A. D. Dayan and M. I. Stokes, Brit. Med. J., 2, 374 ; 1972) suggest that SSPE may be a model of temperate viral infections in humans, possibly an important group

conditions the infected cells have some of the properties characteristic of transformed cells.

The complete replication of Herpesvirus hominis in cultures of human lung cells results in cell lysis, and to prevent this Darai and Munk raised the temperature of the culture 1 hour after infection from $37^{\circ}$ to $42^{\circ} \mathrm{C}$. Cells which survived infection for eight days at $42^{\circ} \mathrm{C}$ were then returned to $37^{\circ} \mathrm{C}$ and repeatedly subcultured. Several cell lines were established and these cells were tested in a variety of ways.

The cells all proved to contain a Herpesvirus hominis antigen in the cytoplasm; to tend to form syncytia as a result of cell fusion apparently promoted by a viral-coded cell surface like a protein; to resist superinfection and to survive longer in culture than control uninfected cells. These properties suggest that the cells may have been transformed. 